\title{
Hook Length Formulas for Trees by Han's Expansion
}

\author{
William Y.C. Chen ${ }^{1}$, Oliver X.Q. Gao ${ }^{2}$ and Peter L. Guo ${ }^{3}$ \\ Center for Combinatorics, LPMC-TJKLC \\ Nankai University, Tianjin 300071, P.R. China \\ ${ }^{1}$ chen@nankai.edu.cn, ${ }^{2}$ oliver@cfc.nankai.edu.cn, ${ }^{3}$ lguo@cfc.nankai.edu.cn
}

Submitted: Mar 19, 2009; Accepted: May 9, 2009; Published: May 15, 2009

Mathematics Subject Classifications: 05A15, 05A19

\begin{abstract}
Recently Han obtained a general formula for the weight function corresponding to the expansion of a series in terms of hook lengths of binary trees. In this paper, we present weight function formulas for $k$-ary trees, plane trees, plane forests, labeled trees and forests. We also find appropriate generating functions which lead to unifications of the hook length formulas due to Du and Liu, Han, Gessel and Seo, and Postnikov.
\end{abstract}

Keywords: hook length formulas for trees, $k$-ary trees, plane trees, labeled trees.

\section{Introduction}

Recently, Han developed an expansion technique for deriving hook length formulas for binary trees. He has shown that given any formal power series $f(x)$ with $f(0)=1$, one can determine the weight function $\rho(n)$ that leads to a hook length formula for binary trees. In this paper, we extend Han's technique and obtain the expansion formulas for $k$-ary trees, plane trees, plane forests, labeled trees and forests. We find appropriate generating functions that can be used to derive new hook length formulas, some of which can be viewed as unifications of the formulas due to Du and Liu [3], Han [6, 7, 8], Gessel and Seo [5].

Let us give a quick review of the background and terminology. For a tree (or a forest) $T$ the hook length of a vertex $u$ of $T$, denoted by $h_{u}$, is the number of descendants of $u$ in $T$ under the assumption that $u$ is counted as a descendant of itself. The hook length multi-set $\mathcal{H}(T)$ of $T$ is defined to be the multi-set of hook lengths of the vertices $u$ of $T$. Clearly, the above definition of hook length applies to all kinds of trees and forests, such as binary trees, plane trees, labeled trees, plane forests, and forests. Figure 1 is an illustration of the multi-set of a tree. 


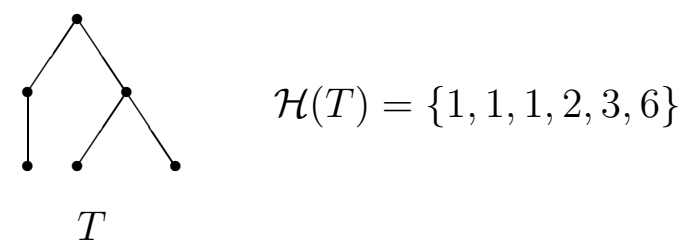

Figure 1: The multi-set of hook lengths of a tree.

Postnikov [11] discovered the following remarkable hook length formula for binary trees

$$
\frac{n !}{2^{n}} \sum_{T} \prod_{h \in \mathcal{H}(T)}\left(1+\frac{1}{h}\right)=(n+1)^{n-1}
$$

where the sum ranges over binary trees with $n$ vertices. Combinatorial proofs of (1.1) have been given by Chen and Yang [2], and Seo [13]. Hook length formulas have been found for $k$-ary trees, plane forests and forests. Du and Liu [3] have obtained the following formulas

$$
\sum_{T} \prod_{h \in \mathcal{H}(T)}\left(a+\frac{1}{h}\right)=\frac{(a+1)}{n !} \prod_{i=1}^{n-1}(k a n+a+1-i(a-k+1)),
$$

where $T$ ranges over $k$-ary trees with $n$ vertices, and

$$
\sum_{F} \prod_{h \in \mathcal{H}(F)}\left(a+\frac{1}{h}\right)=\frac{(a+1)}{n !} \prod_{i=1}^{n-1}((2 n+1)(a+1)-(a+2) i)
$$

where $F$ ranges over plane forests with $n$ vertices. Liu [9] has given a hook length formula for plane forests with a given degree sequence. Gessel and Seo [5] independently discovered (1.2) and (1.3), and obtained the following formula for forests

$$
\sum_{F} \prod_{h \in \mathcal{H}(F)}\left(1+\frac{a}{h}\right)=(a+1) \prod_{i=1}^{n-1}((a+1) n-a i),
$$

where the sum is over forests with $n$ vertices.

Han $[8]$ has found the following formula for binary trees

$$
n ! \sum_{T} \prod_{h \in \mathcal{H}(T)} \frac{(z+h)^{h-1}}{2 h(2 z+h-1)^{h-2}}=z(n+z)^{n-1},
$$

where the sum runs over binary trees with $n$ vertices. Recall that the polynomials $z(n+$ $z)^{n-1}$ are the classical Abel polynomials, see Mullin and Rota [10]. The above formula was first proved by Han [8] by induction, and then it was brought into the framework of his expansion technique [6]. 
Han's expansion technique for binary trees can be described as follows. Denote by $\mathbb{K}[[x]]$ the set of formal power series over some field $\mathbb{K}$. Define the weight function $\rho: \mathbb{N}^{+} \longrightarrow \mathbb{K}$ to be a mapping from the set of positive integers to $\mathbb{K}$. Denote by $B(n)$ the set of binary trees with $n$ vertices. Han [6] has shown that if the following relation holds

$$
1+\sum_{n \geq 1}\left(\sum_{T \in B(n)} \prod_{h \in \mathcal{H}(T)} \rho(h)\right) x^{n}=f(x),
$$

then the weight function $\rho$ is given by

$$
\rho(n)=\frac{\left[x^{n}\right] f(x)}{\left[x^{n-1}\right] f(x)^{2}},
$$

where $\left[x^{n}\right] f(x)$ denotes the coefficient of $x^{n}$ in the formal power series expansion of $f(x)$. The above formula is called the expansion formula for binary trees. Note that each $T \in B(n)(n \geq 1)$ can be decomposed into a triple $\left(T^{\prime}, T^{\prime \prime}, u\right)$, where $T^{\prime} \in B(m)(0 \leq$ $m \leq n-1), T^{\prime \prime} \in B(n-1-m)$ and $u$ is the root of $T$ with hook length $h_{u}=n$. Suppose that (1.6) holds. Then we can deduce that

$$
\begin{aligned}
{\left[x^{n}\right] f(x) } & =\rho(n) \sum_{\left(T^{\prime}, T^{\prime \prime}\right)} \prod_{h \in \mathcal{H}\left(T^{\prime}\right)} \rho(h) \prod_{h \in \mathcal{H}\left(T^{\prime \prime}\right)} \rho(h) \\
& =\rho(n) \sum_{m=0}^{n-1}\left[x^{m}\right] f(x)\left[x^{n-1-m}\right] f(x),
\end{aligned}
$$

which implies (1.7).

For example, let $g(x)$ be defined by the functional equation $g(x)=\exp \{x g(x)\}$ and the relation $f(x)=g(2 x)$. Applying the Lagrange inversion formula (see, Stanley [14, Chapter 5]), we get

$$
\left[x^{n}\right] x g(x)=\frac{1}{n}\left[x^{n-1}\right] \mathrm{e}^{n x}=\frac{n^{n-1}}{n !},
$$

and hence

$$
f(x)=\sum_{n \geq 0}(n+1)^{n-1} \frac{(2 x)^{n}}{n !} .
$$

By Han's expansion formula for binary trees and the Lagrange inversion formula for the expansion of $f(x)^{2}$, the weight function corresponding to the generating function $f(x)$ is given by

$$
\rho(n)=\frac{\left[x^{n}\right] f(x)}{\left[x^{n-1}\right] f(x)^{2}}=1+\frac{1}{n},
$$

which leads to Postnikov's formula (1.1).

This paper is organized as follows. In Section 2, we give a straightforward extension of Han's expansion formula to $k$-ary trees, and find a generating function whose corresponding hook length formula is a unification of several known formulas. In Section 3, 
we consider the expansion formulas for plane trees and plane forests. In Section 4, we present expansion formulas and hook length formulas for labeled trees and forests. To conclude this paper, we raise the question of finding combinatorial interpretations of two hook length formulas for plane forests and labeled forests.

\section{$2 \quad k$-ary trees}

In this section, we begin with a straightforward extension of Han's expansion formula for binary trees to $k$-ary trees. The formula of Yang for $k$-ary trees [16] corresponds to the expansion of $e^{x}$. Moreover, we find a generating function defined by a functional equation which enables us to deduce a hook length formula with one more parameter $z$ compared with the formula independently due to Du and Liu [3], and Gessel and Seo [5].

Recall that a $k$-ary tree is an ordered rooted unlabeled tree where each vertex has exactly $k$ subtrees in linear order where we allow a subtree to be empty. When $k=2$, a $k$-ary tree is called a binary tree. Let $T_{k}(n)$ denote the set of $k$-ary trees with $n$ vertices.

Theorem 2.1 Suppose that we have the following expansion formula for $k$-ary trees

$$
1+\sum_{n \geq 1}\left(\sum_{T \in T_{k}(n)} \prod_{h \in \mathcal{H}(T)} \rho(h)\right) x^{n}=f(x) .
$$

Then the weight function $\rho$ is given by

$$
\rho(n)=\frac{\left[x^{n}\right] f(x)}{\left[x^{n-1}\right] f(x)^{k}}
$$

Example 2.2 Let $f(x)=e^{x}$. Applying (2.1) we get

$$
\rho(n)=\frac{\left[x^{n}\right] e^{x}}{\left[x^{n-1}\right] e^{k x}}=\frac{1}{n k^{n-1}} .
$$

Hence

$$
\sum_{T \in T_{k}(n)} \prod_{h \in \mathcal{H}(T)} \frac{1}{h k^{h-1}}=\frac{1}{n !} .
$$

The above formula (2.2) reduces to the formula of Han [7] for $k=2$. Yang [16] has shown that (2.2) holds for general $k$. Probabilistic and combinatorial proofs of (2.2) have been given by Sagan [12], and Chen, Gao and Guo [1], respectively.

Below is another hook length formula of Han for binary trees.

Theorem $2.3(\operatorname{Han}[6]$, Theorem 6.8) For $n \geq 1$,

$$
\begin{gathered}
\sum_{T \in B(n)} \prod_{h \in \mathcal{H}(T)} \frac{\prod_{i=1}^{h-1}(z a+z+(2 h-i) a+i)}{2 h \prod_{i=1}^{h-2}(2 z a+2 z+(2 h-2-i) a+i)} \\
=\frac{z(a+1)}{n !} \prod_{i=1}^{n-1}(z a+z+(2 n-i) a+i) .
\end{gathered}
$$


The generating function $f(x)$ for the above expansion is given by the following functional equation

$$
g(x)=(a-1) x(1+g(x))^{\frac{2 a}{a-1}}
$$

and the relation

$$
f(x)=(1+g(x))^{z \frac{2 a}{a-1}} .
$$

To extend Han's formula to $k$-ary trees, one needs to find the appropriate extension of the generating function to general $k$.

Theorem 2.4 Let $g(x)$ be defined by the functional equation

$$
g(x)=(a-k) x(1+g(x))^{\frac{k(a-1)}{a-k}},
$$

and $f(x)$ be given by

$$
f(x)=(1+g(x))^{z \frac{a}{a-k}} .
$$

Then the weight function $\rho$ corresponding to the hook length expansion of $f(x)$ for $k$-ary trees is given by

$$
\rho(n)=\frac{\prod_{i=1}^{n-1}(z a+k(a-1) n-i(a-k))}{k n \prod_{i=1}^{n-2}(k z a+k(a-1)(n-1)-i(a-k))},
$$

which implies

$$
\begin{aligned}
\sum_{T \in T_{k}(n)} & \prod_{h \in \mathcal{H}(T)} \frac{\prod_{i=1}^{h-1}(z a+k(a-1) h-i(a-k))}{k h \prod_{i=1}^{h-2}(k z a+k(a-1)(h-1)-i(a-k))} \\
= & \frac{z a}{n !} \prod_{i=1}^{n-1}(z a+k(a-1) n-i(a-k)) .
\end{aligned}
$$

Proof. By the Lagrange inversion formula we obtain

$$
\begin{aligned}
{\left[x^{n}\right] f(x) } & =\frac{1}{n}\left[x^{n-1}\right] z \frac{a}{a-k}(1+x)^{z \frac{a}{a-k}-1}(a-k)^{n}(1+x)^{\frac{k(a-1) n}{a-k}} \\
& =\frac{z a(a-k)^{n-1}}{n !} \prod_{i=0}^{n-2}\left(z \frac{a}{a-k}-1+\frac{k(a-1) n}{a-k}-i\right) \\
& =\frac{z a}{n !} \prod_{i=1}^{n-1}(z a+k(a-1) n-i(a-k)) .
\end{aligned}
$$

Note that $\left[x^{n}\right] f(x)^{k}$ can be easily derived from $\left[x^{n}\right] f(x)$ by substituting $z$ with $k z$. Consequently,

$$
\left[x^{n}\right] f(x)^{k}=\frac{k z a}{n !} \prod_{i=1}^{n-1}(k z a+k(a-1) n-i(a-k)) .
$$


By Theorem 2.1, we obtain the weight function (2.4). This completes the proof.

The formula (2.5) can be viewed as a unification of several known formulas. Setting $k=2$ and substituting $a$ with $a+1$, we obtain Han's formula (2.3). Setting $z=1$ in (2.5) gives

$$
\sum_{T \in T_{k}(n)} \prod_{h \in \mathcal{H}(T)}\left(a-1+\frac{1}{h}\right)=\frac{a}{n !} \prod_{i=1}^{n-1}(k(a-1) n+a-i(a-k)),
$$

which is equivalent to the formula (1.2) derived independently by Du and Liu [3], and Gessel and Seo [5]. Setting $a=k$ and $z=1$ in (2.5), we obtain

$$
\sum_{T \in T_{k}(n)} \prod_{h \in \mathcal{H}(T)}\left(k-1+\frac{1}{h}\right)=\frac{k^{n}((k-1) n+1)^{n-1}}{n !},
$$

which is an extension of Postnikov's hook formula (1.1) to $k$-ary trees. Setting $a=k$ in (2.5), we arrive at the following extension of Han's formula (1.5) to $k$-ary trees.

Theorem 2.5 For $n \geq 1$,

$$
n ! \sum_{T \in T_{k}(n)} \prod_{h \in \mathcal{H}(T)} \frac{(z+(k-1) h)^{h-1}}{k h(k z+(k-1)(h-1))^{h-2}}=z(z+(k-1) n)^{n-1} .
$$

Recall that the polynomials $z(z+(k-1) n)^{n-1}$ are also Abel polynomials (see [10]). Letting $a \rightarrow \infty$ in (2.5), we are led to the following formula.

Theorem 2.6 For $n \geq 1$,

$$
\sum_{T \in T_{k}(n)} \prod_{h \in \mathcal{H}(T)} \frac{\prod_{i=1}^{h-1}(k h+z-i)}{k h \prod_{i=1}^{h-2}(k h+k(z-1)-i)}=\frac{z}{n !} \prod_{i=1}^{n-1}(k n+z-i) .
$$

\section{$3 \quad$ Plane trees and plane forests}

In this section, we derive hook length expansion formulas for plane trees and plane forests, and we find certain generating functions which lead to several hook length formulas. Some known formulas can be brought into the framework of the expansion technique.

A plane tree is a rooted unlabeled tree in which the subtrees, assumed to be nonempty, of each vertex are arranged in linear order. A plane forest is a forest of nonempty plane trees which are linearly ordered. Let $P T(n)$ (resp., $P F(n)$ ) denote the set of plane trees (resp., plane forests) with $n$ vertices. 
Theorem 3.1 Suppose that the following expansion formula holds for plane trees

$$
\sum_{n \geq 1}\left(\sum_{T \in P T(n)} \prod_{h \in \mathcal{H}(T)} \rho(h)\right) x^{n}=f(x) .
$$

Then the weight function $\rho$ is given by

$$
\rho(n)=\frac{\left[x^{n}\right] f(x)}{\left[x^{n-1}\right] \frac{1}{1-f(x)}}, \quad n \geq 1 .
$$

Proof. For a plane tree $T$ with $n(n \geq 2)$ vertices we can construct a $j$-tuple $\left(T_{1}, T_{2}, \ldots, T_{j}\right)$ $(j \geq 1)$ by deleting the root $u$ of $T$, where $T_{i} \in P T\left(m_{i}\right)\left(m_{i}>0\right)$ and $\sum_{i=1}^{j} m_{i}=n-1$. Let $f(n)=\left[x^{n}\right] f(x)$. We see that

$$
\begin{aligned}
f(n) & =\rho\left(h_{u}\right) \sum_{j \geq 1} \sum_{\left(T_{1}, T_{2}, \ldots, T_{j}\right)} \prod_{i=1}^{j} \prod_{h \in \mathcal{H}\left(T_{i}\right)} \rho(h) \\
& =\rho(n) \sum_{j \geq 1} \sum_{m_{1}+\cdots+m_{j}=n-1} f\left(m_{1}\right) f\left(m_{2}\right) \cdots f\left(m_{j}\right)=\rho(n)\left[x^{n-1}\right] \frac{1}{1-f(x)} .
\end{aligned}
$$

Since $\rho(1)=f(1)$, we arrive at $(3.1)$.

We proceed to employ (3.1) to derive hook length formulas for plane trees.

Example 3.2 Let $f(x)$ be defined by

$$
f^{\prime}(x)=\frac{1}{1-f(x)}
$$

with $f(0)=0$. The solution of the above differential equation is given by

$$
f(x)=\sum_{n \geq 1}(2 n-3) ! ! \frac{x^{n}}{n !},
$$

where $n ! !=n(n-2)(n-4) \cdots$ for $n$ odd and $(-1) ! !=1$. By (3.1) we have $\rho(n)=\frac{1}{n}$ so that the following hook length formula holds

$$
n ! \sum_{T \in P T(n)} \prod_{h \in \mathcal{H}(T)} \frac{1}{h}=(2 n-3) ! !
$$

For a plane tree $T$ with $n$ vertices, it is well-known that the number of ways to label the vertices of $T$ with $\{1,2, \ldots, n\}$, such that the labeling of each vertex is less than the labelings of its descendants, is equal to $\frac{n !}{\prod_{h \in \mathcal{H}(T)} h}$, see [5]. On the other hand, $(2 n-3)$ !! equals the number of increasing plane trees on $n$ vertices, see, e.g., [4]. 
Theorem 3.3 For $n \geq 1$, we have

$$
n ! \sum_{T \in P T(n)} \prod_{h \in \mathcal{H}(T)}\left(1-\frac{1}{h}\right)^{h-1}=(n-1)^{n-1} .
$$

Proof. Let

$$
f(x)=\sum_{n \geq 1}(n-1)^{n-1} \frac{x^{n}}{n !} .
$$

It is known that (see, Stanley [14, P. 43])

$$
\frac{1}{1-f(x)}=\sum_{n \geq 0}(n+1)^{n-1} \frac{x^{n}}{n !} .
$$

By Theorem 3.1, we find

$$
\rho(n)=\frac{(n-1)^{n-1}}{n^{n-1}}=\left(1-\frac{1}{n}\right)^{n-1},
$$

which implies (3.3).

We next consider hook length formulas for plane forests.

Theorem 3.4 Suppose that the following expansion formula holds for plane forests

$$
1+\sum_{n \geq 1}\left(\sum_{F \in P F(n)} \prod_{h \in \mathcal{H}(F)} \rho(h)\right) x^{n}=f(x) .
$$

Then the weight function $\rho$ is given by

$$
\rho(n)=-\frac{\left[x^{n}\right] f(x)^{-1}}{\left[x^{n-1}\right] f(x)} .
$$

Proof. To prove (3.4) we notice that

$$
1+\sum_{n \geq 1}\left(\sum_{F \in P F(n)} \prod_{h \in \mathcal{H}(F)} \rho(h)\right) x^{n}=\left(1-\sum_{n \geq 1}\left(\sum_{T \in P T(n)} \prod_{h \in \mathcal{H}(T)} \rho(h)\right) x^{n}\right)^{-1}
$$

that is,

$$
\sum_{n \geq 1}\left(\sum_{T \in P T(n)} \prod_{h \in \mathcal{H}(T)} \rho(h)\right) x^{n}=1-\left(1+\sum_{n \geq 1}\left(\sum_{F \in P F(n)} \prod_{h \in \mathcal{H}(F)} \rho(h)\right) x^{n}\right)^{-1} .
$$

Utilizing (3.1), it is easy to check (3.4). This completes the proof.

Let us consider the above expansion for the exponential function. 
Example 3.5 Let $f(x)=e^{x}$. Then

$$
\rho(n)=\frac{(-1)^{n+1} \frac{1}{n !}}{\frac{1}{(n-1) !}}=\frac{(-1)^{n+1}}{n},
$$

and hence

$$
1+\sum_{n \geq 1} \sum_{F \in P F(n)} x^{n} \prod_{h \in \mathcal{H}(F)} \frac{(-1)^{h+1}}{h}=e^{x} .
$$

Equating the coefficients of $x^{n}$ yields

$$
\sum_{F \in P F(n)} \frac{n !}{\prod_{h \in \mathcal{H}(F)}(-1)^{h} h}=(-1)^{n},
$$

which can be restated in terms of plane trees

$$
\sum_{T \in P T(n)} \frac{n !}{\prod_{h \in \mathcal{H}(T)}(-1)^{h} h}=-1 .
$$

Note that the above formula (3.5) along with a combinatorial proof is given by Yang [16]. The following theorem is concerned with a hook length formula in connection with the Bernoulli numbers.

Theorem 3.6 Let $B_{n}$ be the $n$th Bernoulli number. Then we have

$$
\sum_{F \in P F(n)} \prod_{h \in \mathcal{H}(F)} B_{h}=\frac{(-1)^{n}}{(n+1) !}
$$

Proof. Let $f(x)=\frac{e^{x}-1}{x}$. By the definition of the Bernoulli numbers, we have

$$
\frac{1}{f(x)}=\frac{x}{e^{x}-1}=\sum_{n \geq 0} B_{n} \frac{x^{n}}{n !},
$$

It follows from (3.4) that $\rho(n)=-B_{n}$. Hence

$$
1+\sum_{n \geq 1} \sum_{F \in P F(n)}(-x)^{n} \prod_{h \in \mathcal{H}(F)} B_{h}=\frac{e^{x}-1}{x} .
$$

Equating the coefficients of $x^{n}$ on both sides of (3.7) gives (3.6).

The following theorem is a unification of several known hook length formulas for plane forests.

Theorem 3.7 Let $g(x)$ be defined by the functional equation

$$
g(x)=(a+1) x(1+g(x))^{\frac{2 a}{a+1}},
$$


and $f(x)$ be given by

$$
f(x)=(1+g(x))^{\frac{z a}{a+1}} .
$$

Then the weight function $\rho$ corresponding to the hook length expansion of $f(x)$ for plane forests is given by

$$
\rho(n)=\frac{\prod_{i=1}^{n-1}((2 n-z) a-(a+1) i)}{n \prod_{i=1}^{n-2}((2 n-2+z) a-(a+1) i)} .
$$

Thus we have

$$
\begin{gathered}
\sum_{F \in P F(n)} \prod_{h \in \mathcal{H}(F)} \frac{\prod_{i=1}^{h-1}((2 h-z) a-(a+1) i)}{h \prod_{i=1}^{h-2}((2 h-2+z) a-(a+1) i)} \\
=\frac{z a}{n !} \prod_{i=1}^{n-1}((2 n+z) a-(a+1) i) .
\end{gathered}
$$

Proof. By the Lagrange inversion formula we find

$$
\begin{aligned}
{\left[x^{n}\right] f(x) } & =\frac{1}{n}\left[x^{n-1}\right] \frac{z a}{a+1}(1+x)^{\frac{z a}{(a+1)}-1}(a+1)^{n}(1+x)^{\frac{2 a n}{a+1}} \\
& =\frac{z a}{n !} \prod_{i=1}^{n-1}((2 n+z) a-(a+1) i) .
\end{aligned}
$$

Substituting $z$ with $-z$ yields

$$
\left[x^{n}\right] f(x)^{-1}=\frac{-z a}{n !} \prod_{i=1}^{n-1}((2 n-z) a-(a+1) i) .
$$

By Theorem 3.4, it is easy to verify (3.8) and (3.9).

We now consider some special cases of the above formula (3.9). Taking $z=1$ and substituting $a$ with $a+1$ in (3.9) we get the formula (1.3) of Du and Liu [3], namely,

$$
\sum_{F \in P F(n)} \prod_{h \in \mathcal{H}(F)}\left(a+\frac{1}{h}\right)=\frac{(a+1)}{n !} \prod_{i=1}^{n-1}((2 n+1)(a+1)-(a+2) i) .
$$

Taking $a=1$, (3.9) reduces to the following identity.

Theorem 3.8 For $n \geq 1$,

$$
\sum_{F \in P F(n)} \prod_{h \in \mathcal{H}(F)} \frac{\prod_{i=1}^{h-1}(2 h-z-2 i)}{h \prod_{i=2}^{h-1}(2 h+z-2 i)}=\frac{z}{n !} \prod_{i=1}^{n-1}(2 n+z-2 i) .
$$

Letting $a=-1$ in (3.9), we deduce the following formula. 
Theorem 3.9 For $n \geq 1$,

$$
\sum_{F \in P F(n)} \prod_{h \in \mathcal{H}(F)} \frac{(2 h-z)^{h-1}}{h(2 h-2+z)^{h-2}}=\frac{z}{n !}(2 n+z)^{n-1} .
$$

Setting $z=2$ in (3.11), we get a formula equivalent to (3.3).

Theorem 3.10 For $n \geq 1$,

$$
n ! \sum_{F \in P F(n)} \prod_{h \in \mathcal{H}(F)}\left(1-\frac{1}{h}\right)^{h-1}=(n+1)^{n-1} .
$$

Notice that the right hand side of (3.12) is equal to the number of forests with $n$ vertices. When $a$ tends to infinity, (3.9) leads to the following identity.

Theorem 3.11 For $n \geq 1$,

$$
\sum_{F \in P F(n)} \prod_{h \in \mathcal{H}(F)} \frac{(2 h-z-1)_{h-1}}{h(2 h+z-3)_{h-2}}=\frac{z}{n !}(2 n+z-1)_{n-1},
$$

where $(x)_{n}=x(x-1) \cdots(x-n+1)$ stands for the falling factorial.

\section{Labeled trees and forests}

In this section, we give the expansion formula for labeled trees and forests and derive several new hook length formulas. In particular, we obtain a unified hook length formula which includes the formula (1.4) obtained by Gessel and Seo [5] as a special case. Let $T(n)$ (resp., $F(n))$ denote the set of labeled trees (resp., forests) with $n$ vertices.

Theorem 4.1 Suppose that the following expansion formula holds for labeled trees

$$
\sum_{n \geq 1}\left(\sum_{T \in T(n)} \prod_{h \in \mathcal{H}(T)} \rho(h)\right) \frac{x^{n}}{n !}=f(x)
$$

Then the weight function $\rho$ is given by

$$
\rho(n)=\frac{\left[x^{n}\right] f(x)}{\left[x^{n-1}\right] e^{f(x)}}
$$

Proof. For a labeled tree $T$ with $n(n \geq 2)$ vertices, let $u$ be the root of $T$. Let $\left\{T_{1}, T_{2}, \ldots, T_{j}\right\}(j \geq 1)$ be the set of subtrees of $u$, and let $B_{i}$ be the underlying set 
of $T_{i}$. Keep in mind that $\left\{B_{1}, \ldots, B_{j}\right\}$ is a partition of $[n]-\{u\}$. Set $f(n)=\left[x^{n}\right] f(x)$. Since there are $n$ choices for $u$, we find that

$$
f(n)=\rho(n) n \sum_{j \geq 1} \sum_{\left\{B_{1}, \ldots, B_{j}\right\}} f\left(\# B_{1}\right) \cdots f\left(\# B_{j}\right),
$$

where $\left\{B_{1}, \ldots, B_{j}\right\}$ ranges over all partitions of $[n-1]$ with $j$ blocks and $\# B_{i}$ denotes the cardinality of $B_{i}$. From the exponential formula (see, e.g., Stanley [14]) it follows that

$$
\sum_{j \geq 1} \sum_{\left\{B_{1}, \ldots, B_{j}\right\}} f\left(\# B_{1}\right) \cdots f\left(\# B_{j}\right)=\left[\frac{x^{n-1}}{(n-1) !}\right] e^{f(x)} .
$$

Since $\rho(1)=f(1)$, we conclude that (4.1) is true for $n \geq 1$.

Theorem 4.2 Let $B_{n}$ be the $n$-th Bell number; that is, the number of partitions of $[n]$. Then we have

$$
\sum_{T \in T(n)} \prod_{h \in \mathcal{H}(T)} \frac{1}{h B_{h-1}}=1
$$

Proof. Let $f(x)=e^{x}-1$. It is well-known that

$$
e^{f(x)}=\sum_{n \geq 0} B_{n} \frac{x^{n}}{n !} .
$$

In view of (4.1), we get

$$
\rho(n)=\frac{1}{n B_{n-1}}
$$

as desired.

Theorem 4.3 For $n \geq 1$, we have

$$
\sum_{T \in T(n)} \prod_{h \in \mathcal{H}(T)} \frac{1}{h}=(n-1) !
$$

Proof. Let

$$
f(x)=\ln \frac{1}{1-x} .
$$

Clearly, $e^{f(x)}=\frac{1}{1-x}$. By (4.1) we see that $\rho(n)=\frac{1}{n}$. This completes the proof.

The next theorem gives the hook length expansion formula for forests.

Theorem 4.4 Suppose that the following expansion formula holds

$$
1+\sum_{n \geq 1}\left(\sum_{F \in F(n)} \prod_{h \in \mathcal{H}(F)} \rho(h)\right) \frac{x^{n}}{n !}=f(x) .
$$

Then the weight function $\rho$ is given by

$$
\rho(n)=\frac{\left[x^{n}\right] \ln f(x)}{\left[x^{n-1}\right] f(x)} .
$$


Proof. By the exponential formula, we obtain

$$
f(x)=1+\sum_{n \geq 1}\left(\sum_{F \in F(n)} \prod_{h \in \mathcal{H}(F)} \rho(h)\right) \frac{x^{n}}{n !}=\exp \left\{\sum_{n \geq 1}\left(\sum_{T \in T(n)} \prod_{h \in \mathcal{H}(T)} \rho(h)\right) \frac{x^{n}}{n !}\right\} .
$$

Taking logarithm on both sides yields

$$
\ln f(x)=\sum_{n \geq 1}\left(\sum_{T \in T(n)} \prod_{h \in \mathcal{H}(T)} \rho(h)\right) \frac{x^{n}}{n !} .
$$

Combining the above relation and the expansion formula (4.1) for labeled rooted trees, we get (4.3).

Theorem 4.5 For $n \geq 1$,

$$
\frac{1}{n !} \sum_{F \in F(n)} \prod_{h \in \mathcal{H}(F)} \frac{2(2 h-2) ! !}{h(2 h-3) ! !}=\left(\begin{array}{c}
2 n \\
n
\end{array}\right) .
$$

Proof. Let

$$
f(x)=\frac{1}{\sqrt{1-4 x}}=\sum_{n \geq 0}\left(\begin{array}{c}
2 n \\
n
\end{array}\right) x^{n}
$$

Applying (4.3) we find

$$
\rho(n)=\frac{2(2 n-2) ! !}{n(2 n-3) ! !}
$$

as desired.

The following formula is a unification of several known hook length formulas for forests.

Theorem 4.6 Let $g(x)$ be defined by the functional equation

$$
g(x)=x(a-1)(1+g(x))^{\frac{a}{a-1}},
$$

and $f(x)$ be given by

$$
f(x)=(1+g(x))^{\frac{z a}{a-1}} .
$$

Then the weight function $\rho$ corresponding to the hook length expansion of $f(x)$ for forests is given by

$$
\rho(n)=\frac{\prod_{i=1}^{n-1}(a n-(a-1) i)}{n \prod_{i=1}^{n-2}(a(n-1+z)-(a-1) i)},
$$

and hence

$$
\begin{aligned}
\sum_{F \in F(n)} & \prod_{h \in \mathcal{H}(F)} \frac{\prod_{i=1}^{h-1}(a h-(a-1) i)}{h \prod_{i=1}^{h-2}(a(h-1+z)-(a-1) i)} \\
= & z a \prod_{i=1}^{n-1}(a(n+z)-(a-1) i) .
\end{aligned}
$$


Proof. By the Lagrange inversion formula we get

$$
\begin{aligned}
{\left[x^{n}\right] f(x) } & =\frac{1}{n}\left[x^{n-1}\right] \frac{z a}{a-1}(1+x)^{\frac{z a}{a-1}-1}(a-1)^{n}(1+x)^{\frac{a n}{a-1}} \\
& =\frac{z a}{n !} \prod_{i=1}^{n-1}(a(n+z)-(a-1) i)
\end{aligned}
$$

and

$$
\begin{aligned}
{\left[x^{n}\right] \ln f(x) } & =\frac{1}{n}\left[x^{n-1}\right] \frac{z a}{a-1}(1+x)^{-1}(a-1)^{n}(1+x)^{\frac{a n}{a-1}} \\
& =\frac{z a}{n !} \prod_{i=1}^{n-1}(a n-(a-1) i) .
\end{aligned}
$$

By Theorem 4.4, it is easy to verify (4.5), and so we obtain (4.6).

Setting $z=1$ in (4.6), we obtain the following formula equivalent to (1.4) derived by Gessel and Seo [5]:

$$
\sum_{F \in F(n)} \prod_{h \in \mathcal{H}(F)}\left(1+\frac{a-1}{h}\right)=a \prod_{i=1}^{n-1}(a n-(a-1) i) .
$$

Setting $a=1$ in (4.6), we have the following formula.

Theorem 4.7 For $n \geq 1$,

$$
\sum_{F \in F(n)} \prod_{h \in \mathcal{H}(F)}\left(\frac{h}{h-1+z}\right)^{h-2}=z(n+z)^{n-1} .
$$

Letting $a \rightarrow \infty$ in (4.6), we are led to the following identity.

Theorem 4.8 For $n \geq 1$,

$$
\sum_{F \in F(n)} \prod_{h \in \mathcal{H}(F)} \frac{(h-1) !}{h(h-2+z)_{h-2}}=z(n+z-1)_{n-1} .
$$

Setting $z=1$ in (4.8) yields a formula which is equivalent to (4.2).

Theorem 4.9 For $n \geq 1$,

$$
\sum_{F \in F(n)} \prod_{h \in \mathcal{H}(F)} \frac{1}{h}=n !
$$


Thomas [15] has found a simple combinatorial interpretation of the above formula (4.9). Let us rewrite (4.9) in the following form

$$
\sum_{F \in F(n)} n ! \prod_{h \in \mathcal{H}(F)} \frac{1}{h}=(n !)^{2} .
$$

Recall that given a labeled forest $F, \frac{n !}{\prod_{h \in \mathcal{H}(F)} h}$ equals the number of increasing labelings of $F$, see, Gessel and Seo [5]. Thus, the sum on the left hand side of (4.10) equals the number of labeled forests with additional increasing labelings. Formally speaking, any vertex of such a forest is associated with a pair of labels $\left(v_{i}, u_{i}\right)$. We assume that the labels $u_{i}$ correspond to an increasing forest. We now establish a one-to-one correspondence between such forests and pairs $(\pi, w)$, where $\pi=\pi_{1} \pi_{2} \cdots \pi_{n}$ is a permutation on $[n]$ and $w=w_{1} w_{2} \cdots w_{n}$ is a sequence satisfying $1 \leq w_{i} \leq i$ for $1 \leq i \leq n$.

We first construct a forest with an increasing labeling from a given pair $(\pi, w)$. We begin with the vertex with label $\left(\pi_{1}, 1\right)$. Then we determine the position of the vertex $\left(\pi_{2}, 2\right)$. We add $\left(\pi_{2}, 2\right)$ as the child of $\left(\pi_{1}, 1\right)$ if $w_{2}=1$ and as the root of a new branch if $w_{2}=2$. Suppose we have obtained a forest with an increasing labeling by inserting $\pi_{1}, \ldots, \pi_{i}$. Then $\left(\pi_{i+1}, i+1\right)$ is added as a child of the vertex $\left(\pi_{w_{i+1}}, w_{i+1}\right)$ if $w_{i+1} \leq i$ and as the root of a new branch if $w_{i+1}=i+1$. Iterating the above procedure, one obtains a forest with an increasing labeling.

The reverse process can be described as follows. For a forest with an increasing labeling, the vertex $\left(v_{n}, n\right)$ is either an isolated vertex or a leaf. If $\left(v_{n}, n\right)$ is an isolated vertex, then let $\pi_{n}=v_{n}$ and $w_{n}=n$; otherwise, let $\pi_{n}=v_{n}$ and $w_{n}=j$ where $\left(v_{j}, j\right)$ is the parent of $\left(v_{n}, n\right)$. We continue to consider the forest obtained by deleting $\left(v_{n}, n\right)$. Similarly, we can determine $\pi_{n-1}$ and $w_{n-1}$. Iterating this process gives a pair $(\pi, w)$. This completes the combinatorial proof of (4.10).

Setting $z=2$ in (4.8), we obtain the following formula.

Theorem 4.10 For $n \geq 1$,

$$
\sum_{F \in F(n)} \prod_{h \in \mathcal{H}(F)} \frac{1}{h^{2}}=\frac{(n+1) !}{2^{n}} .
$$

It would be interesting to find combinatorial interpretations of the formulas (3.12) and (4.11).

Acknowledgments. We would like to thank Guo-Niu Han and Hugh Thomas for valuable suggestions. This work was supported by the 973 Project, the PCSIRT Project of the Ministry of Education, the Ministry of Science and Technology, and the National Science Foundation of China. 


\section{References}

[1] W.Y.C. Chen, O.X.Q. Gao and P.L. Guo, Combinatorial proofs of Han's hook length formulas for trees, preprint, 2009.

[2] W.Y.C. Chen and L.L.M. Yang, On Postnikov's hook length formula for binary trees, European J. Combin. 29 (2008), 1563-1565.

[3] R.R.X. Du and F. Liu, $(k, m)$-Catalan numbers and hook length polynomials for plane trees, European J. Combin. 28 (2007), 1312-1321.

[4] I.M. Gessel, B. Sagan and Y.N. Yeh, Enumeration of trees by inversions, J. Graph Theory, 19 (1995), 435-459.

[5] I.M. Gessel and S. Seo, A Refinement of Cayley's formula for trees, Electron. J. Combin. 11 (2) (2006), R27.

[6] G.-N. Han, Discovering new hook length formulas by expansion technique, Electron. J. Combin. 15 (1) (2008), R133.

[7] G.-N Han, New hook length formulas for binary trees, Combinatorica, to appear.

[8] G.-N Han, Yet another generalization of Postnikov's hook length formula for binary trees, SIAM J. Discrete Math, to appear.

[9] F. Liu, Hook length polynomials for plane forests of a certain type, Ann. Combin., to appear.

[10] R. Mullin and G.-C. Rota, On the foundations of combinatorial theory III. Theory of binomial enumeration, B. Harris (ed.), Graph Theory and Its Applications, pp. 167-213, Academic Press, 1970.

[11] A. Postnikov, Permutohedra, associahedra, and beyond, Int. Math. Res. Notices (2009) 2009, 1026-1106.

[12] B.E. Sagan, Probabilistic proofs of hook length formulas involving trees, Sém. Lothar. Combin., Special issue dedicated to the memory of Pierre Leroux, 61A (2009), Art. B61Aa.

[13] S. Seo, A combinatorial proof of Postnikov's identity and a generalized enumeration of labeled trees, Electron. J. Combin. 11 (2), N3.

[14] R.P. Stanley, Enumerative Combinatorics, Vol. 2, Cambridge University Press, Cambridge, UK, 1999.

[15] H. Thomas, Private communication, 2009.

[16] L.L.M. Yang, Generalizations of Han's hook length identities, arXiv:math.CO/0805. 0109. 